\title{
Recognizing the subject exposure from the EEG signals with artificial neural networks
}

\author{
Sašo Pavlič \\ University of Maribor, Faculty \\ of Electrical Engineering and \\ Computer Science \\ Koroška cesta 46 \\ Maribor, Slovenia \\ saso.pavlic@student.um.si
}

\author{
Sašo Karakatič \\ University of Maribor, Faculty \\ of Electrical Engineering and \\ Computer Science \\ Koroška cesta 46 \\ Maribor, Slovenia \\ saso.karakatic@um.si
}

\begin{abstract}
The paper presents the analysis of Electroencephalography (EEG) brain waves from the Emotiv Insight device with machine learning, more specifically neural networks. The captured EEG data represents the input data into a machine learning model, which was used to determine when and where the required patterns appear. The experiment of the developed method of capturing data and model usage was carried out by exposing the test subject to the alternating selected images and capturing the EEG brain waves with the Emotiv Insight device. The captured EEG data served as a dataset from which the artificial neural network classification model learnt to successfully recognize when a test subject was exposed to one type of image and when to another. Convolutional and recurrent neural network models were constructed and tested to evaluate the performance of recognition of subject exposal.
\end{abstract}

\section{Keywords}

Electroencephalography, Neural Networks, Machine Learning, EEG signals

\section{INTRODUCTION}

Recently the analysis of Electroencephalography (EEG) data has gained much attention with the development of new measuring techniques and the advancement of the machine learning algorithms and methods. Simpraga et al. [1] proposed a machine learning technique for detection of cholinergic and Alzheimer's disease. Boashash and Ouelha presented a method with machine learning for detection of seizures of newborns [2]. Vanegas et al. presented a machine learning method for detecting of Parkinson's disease [3]. In the same manner, our research was focused on the analysis of EEG data and recognition of subject exposures based on the EEG data with machine learning. Recognizing the simple subject visual exposures can be used in various fields, from user experience, marketing and numerous psychology experiments [4], but there is a lack of research demonstrating the usage of neural networks for this case. This paper intends to fill in this gap.

\subsection{Overview of EEG}

There are four different EEG frequency bands.

Delta $(0.5-3 \mathrm{~Hz})$

The lowest frequency of brain waves moving below $3 \mathrm{~Hz}$ occurs primarily in deep sleep. This frequency is prevalent in infants up to one year of age. It is also present between the 3rd and 4th stages of sleep. Delta waves are reduced in very intense concentration and when we use our thinking processes very actively. Interest is found in individuals who have problems with comprehension and learning. They naturally magnify delta waves; when they want to gather, they fail to reduce it. It is for this reason that the phenomena limit their ability to direct concentration and learning. In this state, we find the brain in a locked-in repetitive state, because in that state we dream or are drowsy.

\section{Theta (3-8 Hz)}

Also classified as slower brain activity. The connection can be made with creativity, intuition, daydreaming and fantasy. It also covers memories, emotions and feelings. Theta waves can be expressed through prayer, meditation and spiritual capture. It can be said to occur between waking consciousness and sleeping. When theta wave is optimal, it allows for flexible and complex behavior structures such as learning and remembering. The imbalance of these waves may indicate illness or stress present.

\section{Alfa $(8-12$ Hz)}

Normal alpha status allows for fast and efficient task management. In this condition, most people feel relaxed and calm. You could say that this wave is like a bridge between the conscious and the unconscious. The alpha state is associated with extraversion, creativity (when solving a problem or listening), and having mental work. When the alpha waves are at the optimum range, we experience well-being, see the world positively, and feel a sense of calm. This situation is one of the most important when learning and using information already learned, such as work and education.

\section{Beta $(12-38 \mathrm{~Hz})$}

The ripple is typical of "fast" activities. This wave is taken as a normal rhythm and is the dominant wave when the person is collected or upset with the eyes open. Waves also occur in listening, thinking, analytical problem solving, decision making, information processing, etc. Because of its relatively wide range, this wave is divided into low, medium and high beta waves.

\section{Gama (38-42 Hz)}

It is a unique frequency wave that is present in all parts of our brains. When they have to process certain information from different parts, it is precisely the $40 \mathrm{~Hz}$ frequency that combines the necessary brain regions for simultaneous processing of data. When we remember something well, it's at $40 \mathrm{~Hz}$ activity.

\section{READING EEG AND ANALYSIS}

For recording the brainwaves we have been using BCI Emotiv Insight, which has the excellent API for accessing that data directly from the device using Bluetooth protocol. With the API we managed to get the raw EEG values for each sensor out of five. That data was received in JSON format. Next, to the values from the device, we have been also adding the marker which was the 
indicator for us to know which type of the image was user looking when specific values from the channel were recorded. In the end, our recorded dataset had the following structure:

- ID,

- TIMESTAMP,

- RAW_CQ (raw value of the quality of the signal),

- Values from the electrodes in $\mathrm{mV}$ for each location on the head (Af3, T7, P7, T8, Af4) (semanticscholar, 2019),

- $\operatorname{EXPOSURE}(0=$ nature images, $1=$ food images $)$.

First, we created the desktop version of the application, which was used as a bridge between the connecting the device with the PC and the presentation media to display the images to the user. Application's basic workflow was that first, we established the connection between the device and the PC, we had to choose the type of image dataset, and the interval of the presentation.

\section{IMPLEMENTATING THE EEG ANALYSIS FRAMEWORK}

With all that set we run the recording and first displayed to the user the blank screen, just for calibrating the data when a user is having closed/open eyes and watching the monitor.

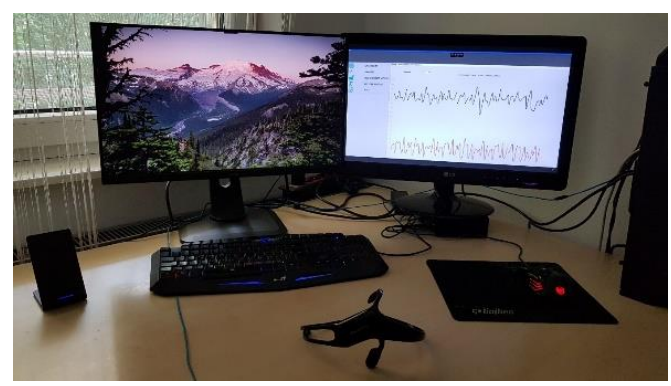

Figure 1. The experimental setup.

After $30 \mathrm{sec}$ of each, the pictures (Figure 2) from the selected dataset started to switch in the selected interval. Our recordings lasted maximum to 30 minutes, depending on the calmness, relaxation of the user. It was really hard to stay concentrated for a while, just looking at the pictures, without thinking and moving much.

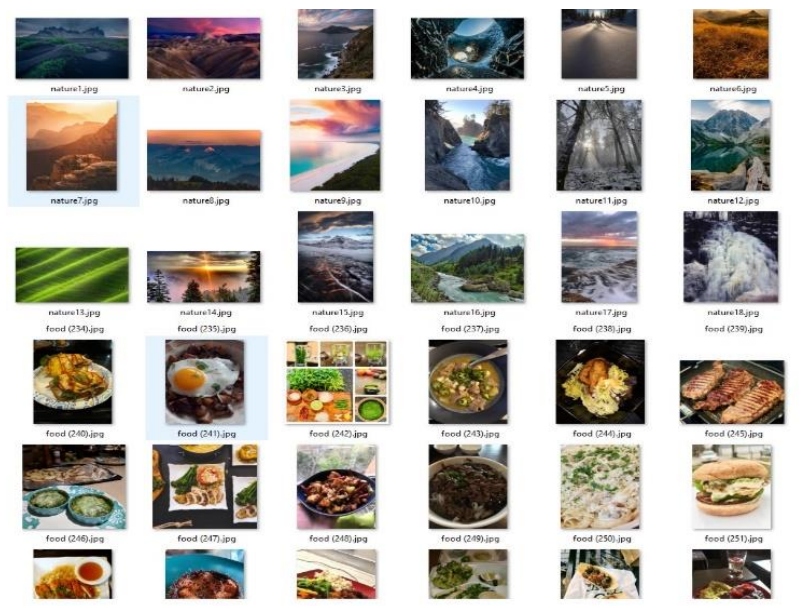

Figure 2. The picture sets for the experiment.
We repeated the recordings on the same user for multiple hours for each type of dataset. Our recording room was isolated from the outside noise and with the constant lighting and minimalism of the objects in the room. We knew that we have to eliminate as much as possible distractions to get reliable recordings from the consumerbased device. All that data was saved in a CSV format per recording, on the interval of $100 \mathrm{~ms}$ (this is the interval of each read of data made by the device).

\begin{tabular}{|llllllllll|}
\hline ID & \multicolumn{2}{|c|}{ Timestamp Raw_value } & Af3 & T7 & P7 & T8 & Af4 & Marker \\
\hline $\mathbf{0}$ & 115 & 500 & 4279.487 & 4250.256 & 4254.872 & 4225.641 & 4291.795 & 0 \\
\hline $\mathbf{1}$ & 230 & 500 & 4252.821 & 4209.231 & 4249.231 & 4165.641 & 4246.667 & 0 \\
\hline $\mathbf{2}$ & 345 & 1023 & 4267.692 & 4235.897 & 4192.308 & 4167.179 & 4249.231 & 0 \\
\hline $\mathbf{3}$ & 460 & 500 & 4253.333 & 4229.744 & 4276.41 & 4169.744 & 4240.513 & 0 \\
\hline $\mathbf{4}$ & 575 & 1023 & 4262.564 & 4203.077 & 4183.077 & 4165.641 & 4246.154 & 0 \\
\hline
\end{tabular}

Table 1. Collected dataset of EEG signals from Emotive Insight device.

It was a mixture of the recorded values from different datasets. Our entire dataset with the length about 50.000 rows. This was the result of almost 2 hours of recording the brainwaves. We split the list into two groups for having the data for testing and learning process in ML. The ratio was 80:20 for the learning process. With all that ready we continued our work, with creating the artificial neural network model.

\subsection{Analysis with Neural Network}

Python framework Keras helped us to create the following neural model. The type of model we have created is called the sequential model and allows you to create multiple levels one by one. It is limited in that it does not allow you to create models that share layers or have multiple inputs or outputs

In the input level it is required to define the dimension of the data in the first level, because at the beginning the model cannot know what data will come to the input. Input data in our case were the values from the electrodes (Af3, T7, P7, T8, Af4).

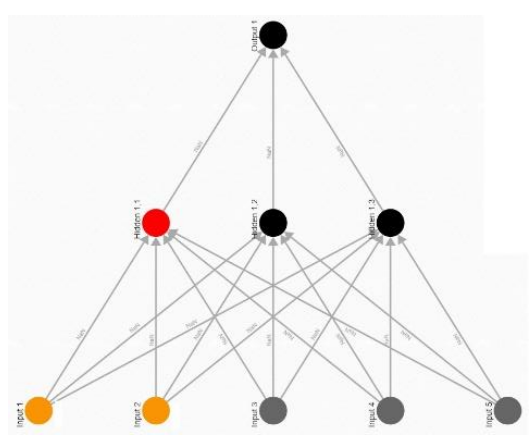

Figure 3. Construction of neural network model with three layers.

Normal distribution was used to initialize the weights, which initialized the weights according to the function results. The activation parameter was defined with a well-established and relatively simple Relu function in the input and hidden levels of the model. For the output level, however, we had to define a sigmoid function that allows us to get a result between 0 and 1 .

We also added a so-called level dropout among the individual levels, which serves to ensure that randomly selected neurons are omitted during the learning phase. This means that the results of the forward pass activation function are removed, as well as any weight 
change is not applied to the backward pass. All these procedures help to make the model generalizable.

When we had finished defining the model, we still had to compile the model with a function that would define a loss (optimizer) that would be able to update the weights according to the result and a function that would return us the result, with what precision in percent, the model predicted the result with given parameters (weights and bias).

model. compile (loss="binary_crossentropy",

optimizer="adam",

metrics=['accuracy'])

Algorithm 2. Compilation of neural network model.

In the next step, we still had to set the parameters for how the model would learn.

$$
\begin{aligned}
& \text { model.fit(x_train, } \\
& \qquad \begin{array}{l}
\text { y_train, } \\
\text { batch_size=124, } \\
\text { epochs }=1000, \\
\text { validation_data=(x_test, y_test)) }
\end{array}
\end{aligned}
$$

Algorithm 3. Fitting of the neural network model.

As the first two parameters, we sent the data to be provided for learning along with the results, and we also defined the size of the data packets in one epoch. In practice, this meant more or less that we repeatedly started learning with different values of the size of the batch and epoch.

To determine how well our neural model performed, we used the evaluate function, which returns the loss and metric values for the model under test.

We obtained a result from the results, which showed that our model predicted the result to reach an accuracy of $55 \%$, which was not an impressive result.

\subsection{Analysis with Recurrent Neural Network}

We can more easily imagine this type of neural model by thinking about how our thoughts work, they always relate to our previous thoughts, we could say that we never start thinking from scratch. This is also how the recurrent-type neural model (RNN) works, which does not restart at every iteration. Traditional NNs do not do this, which is a disadvantage.

Our brainwaves data read on a given image is like a list in which at a given moment, through different positions (Af3, T7, P7, T8, Af4), we can find out which image was shown to the user. However, these values cannot provide us with high reliability of the result, as there were significantly too many factors present when recording data such as electrode reliability, deconcentrating of the user, sudden movements of the user's head, physiological processes in the body, etc. For these reasons, it might be better to want to get that search result across multiple data records, because within those records we can define a pattern in our data that could give us a more reliable result. Because even in the presence of external factors that interfere with our data reliability, through a large amount of records these values are limited to a given stock of values.

We also used the Keras API to build the RNN model, using the SimpleRNN layer, which is a fully connected RNN, where the output from the previous step is sent back to the input for a new learning step. The RNN model accepts a 3-dimensional input type for the input, and our data is in the 2-dimensional type, the third data will represent the step here. Data transformation was achieved by the numpy.reshape method.
Once we had the data in the required type and form, we started building the RNN model. We used again as a input data values from the electrodes (Af3, T7, P7, T8, Af4).

For which we defined the model type as sequential. We added a SimpleRNN level with 32 dimensions and two Dense levels to the model, the first with 8 dimensions and the output with one. For simplicity, we used activation function Relu.

Finally, when we built the model, we further defined the optimization function as RmsProp which is designed for RNN models and the loss function as the average squared error.

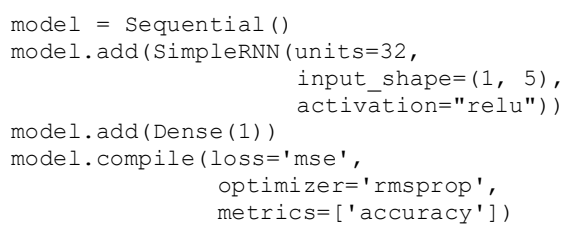

Algorithm 6. Construction of recurrent neural network model.

After learning the model, we got a good start (approx. 62\% accuracy), which was a good starting point for optimizing our model. We have added another Dense level to our model, which should help with the intermediate learning steps, since we want to get better results with the RNN model than with the previous NN, but we need to know that there are many more factors (parameters, hidden levels, understanding the flow of data) that make it difficult to understand and refine the model.

In the end, we obtained an accuracy that defines the accuracy of the result of $80 \%$, which in our case is a satisfactory result, but in practice, unfortunately, it would not be enough to use the model for commercial purposes or for research. Therefore, we decided to change our model by removing the second last level with eight neurons, as it improved the learning result compared to the previous model but still did not succeed in getting a better result. What happened here was that the model became too complex and because of this, the activation functions failed, leading the learning phase to better weight adjustments that would give better learning results.

In the end, our model looked the same as it did at the beginning of defining the model. Here, we then started from the beginning and, before SimpleRNN, inserted a new entry level (Embeding), which transforms positive data with numbers into dense vectors, for example:

$$
[[4],[20]] \rightarrow[[0.25,0.1],[0.6,-0.2]]
$$

This level therefore does not require $3 \mathrm{D}$ data at the beginning, so we also removed the line from our code for converting 2D data into 3D. The output type from Embeding layer is in 3D, which suited us in passing the data to our SimpleRNN layer.

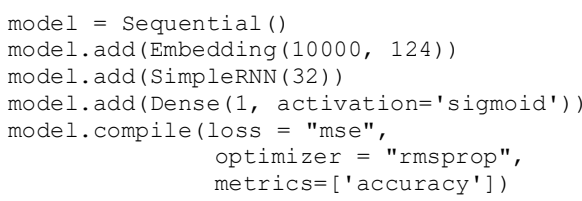

Algorithm 8. The final RNN model.

\section{RESULTS OF THE EXPERIMENT AND CONCLUSION}

In our scenario, we had a collection of data that represented the read values from the BCI device per channel and a marker to indicate 
which image the user observed while reading the values. So we had 6 properties that we split into two lists. The first with values from the device and the second with the marker that represented the result. All of this information was further divided into a learning and test list.

We used two different machine learning models in our process. With the classic NN model, we got worse results because it was obvious that we had too little data despite recording data for hours on the user. Also for this reason NN performed worse because it only changes weights at the end of learning. Meanwhile, the RNN changed weights during the learning step itself, eventually making significantly better predictions, despite the small amount of learning data, as it used internal memory to use the result from the previous output to improve the new one.

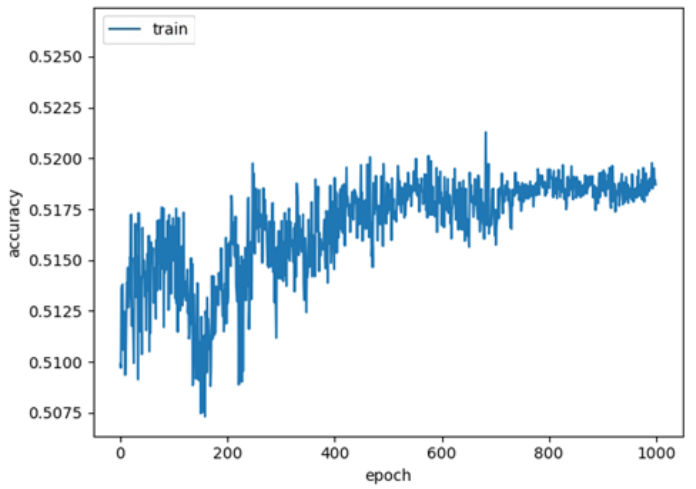

Figure 4. Accuracy through the epochs of the training process for the first simple NN model.

Figure 4 shows how the first simple NN model learned over training process. We can see that the model improved its accuracy in the first half of learning, but towards the end more or less came closer to the same values. If we significantly increased the number of epochs, the result did not improve over time.

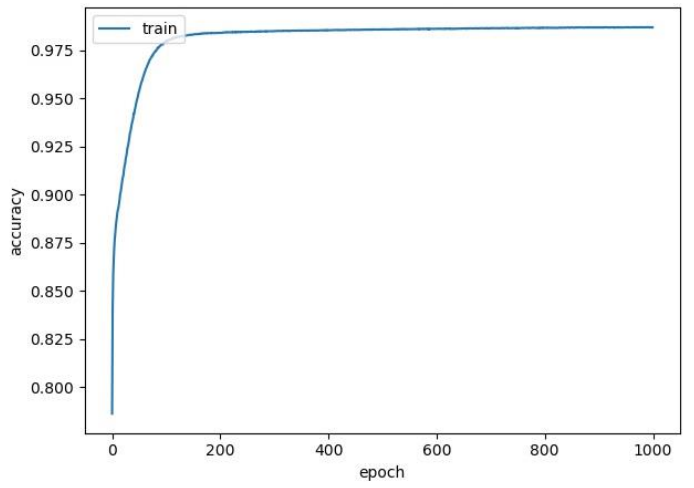

Figure 5. Accuracy through the epochs of the training process for the RNN model.

However, when learning the RNN model (Figure 5), we can see that the accuracy of the model has increased dramatically from the beginning of learning. This change was aided by a new type of data in which the learning process then had a better ability to adjust weights during learning, as well as the RNN model sending the learned state from the previous output to the input, which contributes to the "current" learning. Due to all the running factors, this model performed great compared to a regular $\mathrm{NN}$.

We can also see the loss in the Figure 6 as it declined over time, which meant that our model was getting better at telling the result or the user was looking at pictures of nature or food.

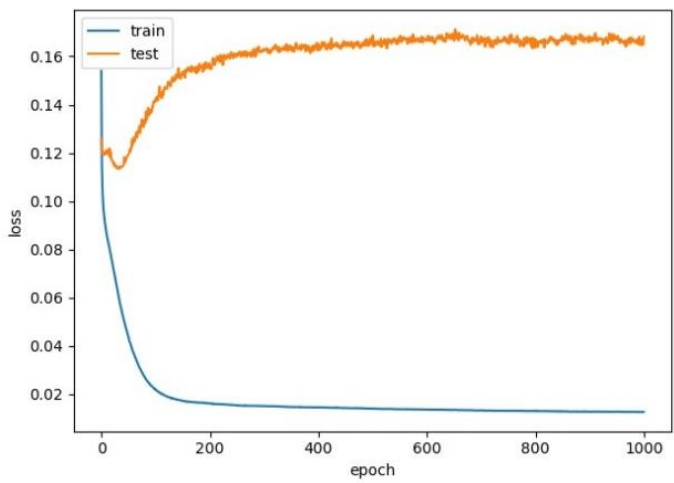

Figure 6. The loss the epochs of the training process for the RNN model.

All these results were still highly dependent on all the external factors that were present in the initial data capture. Initially, the quality and precision of the BCI device must be considered, as it is intended for commercial users and not for professional use, then we must know that user recording is very demanding, and a special gel must be present on the device to help increase electrode conductivity to capture electromagnetic waves, the electrodes should be also as close to the scalp as possible and with as large surface as possible. Another factor is, the mood of the user himself when scanning whether he was always asleep, steady, relaxed, focused are all arguments that should always be the same. All these factors influence the quality of the data and the later classification with machine learning.

\section{ACKNOWLEDGMENTS}

The authors acknowledge financial support from the Slovenian Research Agency (Research Core Funding No. P2-0057).

\section{REFERENCES}

[1] Simpraga, S., Alvarez-Jimenez, R., Mansvelder, H.D., Van Gerven, J.M., Groeneveld, G.J., Poil, S.S. and Linkenkaer-Hansen, K., 2017. EEG machine learning for accurate detection of cholinergic intervention and Alzheimer's disease. Scientific reports, 7(1), p.5775.

[2] Boashash, B. and Ouelha, S., 2016. Automatic signal abnormality detection using time-frequency features and machine learning: A newborn EEG seizure case study. Knowledge-Based Systems, 106, pp.38-50.

[3] Vanegas, M.I., Ghilardi, M.F., Kelly, S.P. and Blangero, A., 2018, December. Machine learning for EEG-based biomarkers in Parkinson's disease. In 2018 IEEE International Conference on Bioinformatics and Biomedicine (BIBM) (pp. 2661-2665). IEEE.

[4] Subha, D.P., Joseph, P.K., Acharya, R. and Lim, C.M., 2010. EEG signal analysis: a survey. Journal of medical systems, 34(2), pp.195-212. 